\title{
SIMPLICIAL VOLUME VIA NORMALISED CYCLES
}

\author{
CLARA LÖH AND MARCO MORASCHINI
}

\begin{abstract}
We show that the Connes-Consani semi-norm on singular homology with real coefficients, defined via s-modules, coincides with the ordinary $\ell^{1}$-semi-norm on singular homology in all dimensions.
\end{abstract}

\section{INTRODUCTION}

Connes and Consani introduced a semi-norm on singular homology via s-modules and established that this semi-norm is equivalent to the $\ell^{1}$-seminorm defined by Gromov [CC20]. Moreover, they proved that their seminorm is equal to the $\ell^{1}$-semi-norm in the case of surfaces [CC20, Theorem 1.4], using a delicate construction specific to surfaces. In this note, we show that the two semi-norms agree in all dimensions, thereby confirming and extending a conjecture of Connes and Consani [CC20, p. 4]:

Theorem 1.1. Let $X$ be a topological space, let $n \in \mathbb{N}$, let $\alpha \in H_{n}(X ; \mathbb{R})$, and let $\lambda \in \mathbb{R}_{>0}$. Then $\|\alpha\|_{1}<\lambda$ if and only if $\alpha$ lies in the image of the canonical map $H_{n}\left(X ;\|H \mathbb{R}\|_{\lambda}\right) \rightarrow H_{n}(X ; \mathbb{R})$.

In particular, the simplicial volume of closed manifolds can also be expressed in terms of homology of s-modules.

As explained by Connes and Consani, in order to show Theorem 1.1]it suffices to prove that the $\ell^{1}$-semi-norm on singular homology can be computed via normalised singular cycles (see Section 2.3):

Proposition 1.2. Let $X$ be a topological space and let $n \in \mathbb{N}$. Then, for all $\alpha \in H_{n}(X ; \mathbb{R})$, we have

$$
\|\alpha\|_{1}=\|\alpha\|_{1}^{\text {norm }} .
$$

In Section 2, we recall basic definitions and notation. The proof of Proposition 1.2 is given in Section 3, based on a symmetrisation construction.

\section{The (NORMALISED) $\ell^{1}$-SEMI-NORM}

2.1. The singular chain complex. Let $n \in \mathbb{N}$ and let $\Delta^{n}$ be the standard $n$-simplex. For $j \in\{0, \cdots, n\}$, we denote by $\iota_{j}^{n}: \Delta^{n-1} \rightarrow \Delta^{n}$ the affine inclusion of the $j$-th facet of $\Delta^{n}$.

Given a topological space $X$, we consider the singular simplicial set $S(X)$ : For $n \in \mathbb{N}$, we have $S_{n}(X):=\operatorname{map}\left(\Delta^{n}, X\right)$ and for $j \in\{0, \ldots, n\}$, the face

Date: May 25, 2020. (c) C. Löh, M. Moraschini 2020. This work was supported by the CRC 1085 Higher Invariants (Universität Regensburg, funded by the DFG).

2020 Mathematics Subject Classification. 55N10, 57N65, 18N50, 18G35, 18G90.

Key words and phrases. simplicial volume, semi-norms on homology, s-modules. 
maps $\partial_{j}: S_{n}(X) \rightarrow S_{n-1}(X)$ are given by

$$
\partial_{j}(\sigma):=\sigma \circ \iota_{j}^{n}
$$

for all $\sigma \in \operatorname{map}\left(\Delta^{n}, X\right)$. The singular chain complex $C_{\bullet}(X ; \mathbb{R})$ with real coefficients is the free $\mathbb{R}$-chain complex associated with $S(X)$.

Furthermore, we have the Moore normalisation $N C_{\bullet}(X ; \mathbb{R})$ of $C_{\bullet}(X ; \mathbb{R})$, given by the submodules

$$
N C_{n}(X ; \mathbb{R}):=\bigcap_{j=0}^{n-1} \operatorname{ker}\left(\partial_{j}\right) \subseteq C_{n}(X ; \mathbb{R})
$$

and the boundary maps $d:=\partial_{n}: N C_{n}(X ; \mathbb{R}) \rightarrow N C_{n-1}(X ; \mathbb{R})$.

Definition 2.1 (normalised chain). A singular chain $c \in C_{n}(X ; \mathbb{R})$ is normalised if it lies in the submodule $N C_{n}(X ; \mathbb{R})$.

2.2. The $\ell^{1}$-semi-norms. We briefly recall Gromov's $\ell^{1}$-semi-norm on singular homology Gro82]: The $\ell^{1}$-norm $\|\cdot\|_{1}$ on $C_{n}(X ; \mathbb{R})$ associated with the basis $S_{n}(X)$ induces a semi-norm on $H_{n}(X ; \mathbb{R})$, the $\ell^{1}$-semi-norm, which we will also denote by $\|\cdot\|_{1}$.

Following Connes and Consani [CC20], one can also endow $H_{n}(X ; \mathbb{R})$ with the semi-norm induced by the $\ell^{1}$-norm on the normalised complex $N C \bullet(X ; \mathbb{R})$ : For $\alpha \in H_{n}(X ; \mathbb{R})$ one sets

$\|\alpha\|_{1}^{\text {norm }}:=\inf \left\{\|c\|_{1} \mid c \in C_{n}(X ; \mathbb{R})\right.$ is a normalised cycle representing $\left.\alpha\right\}$.

Connes and Consani prove that the two semi-norms are equivalent $\mathrm{CC20}$, Lemma 3.4], namely, for every $\alpha \in H_{n}(X ; \mathbb{R})$, we have

$$
\|\alpha\|_{1} \leq\|\alpha\|_{1}^{\text {norm }} \leq \max \left(1,2^{n-1}\right) \cdot\|\alpha\|_{1} .
$$

Proposition 1.2 states that they are in fact equal.

2.3. Deriving Theorem 1.1 from Proposition 1.2. Connes and Consani introduce a filtration of the s-module $H \mathbb{R}$ by a family $\left(\|H \mathbb{R}\|_{\lambda}\right)_{\lambda \in \mathbb{R}>0}$ of sub-s-modules and, for topological spaces $X$, associated singular homology objects $\left(H_{n}\left(X ;\|H \mathbb{R}\|_{\lambda}\right)_{\lambda \in \mathbb{R}>0}\right.$ CC20]. Moreover, these come with canonical maps

$$
\varrho_{n, \lambda}: H_{n}\left(X ;\|H \mathbb{R}\|_{\lambda}\right) \rightarrow H_{n}(X ; \mathbb{R})
$$

to the singular homology of $X$ [C20, Section 3.4]. This filtration defines a semi-norm on $H_{n}(X ; \mathbb{R})$ that is equivalent to $\|\cdot\|_{1}$ [CC20, Corollary 3.6]. More precisely, the image of $\varrho_{n, \lambda}$ coincides with the set of elements $\alpha \in$ $H_{n}(X ; \mathbb{R})$ with $\|\alpha\|_{1}^{\text {norm }}<\lambda[\mathrm{CC} 20$, Theorem 3.5]. Therefore, Theorem 1.1 is a direct consequence of Proposition 1.2 .

\section{Proof of Proposition 1.2}

3.1. Symmetrisation of chains. We recall the symmetrisation map on singular chains, which is given by averaging singular simplices over all vertexpermutations of the standard simplex: In the following, let $X$ be a topological space and $n \in \mathbb{N}$. Let $\Sigma_{n+1}$ denote the symmetric group on $\{0, \ldots, n\}$ and sgn: $\Sigma_{n+1} \rightarrow\{ \pm 1\}$ the sign function. For a map $\pi:\{0, \ldots, k\} \rightarrow\{0, \ldots, n\}$, we write $\Delta(\pi):=[\pi(0), \ldots, \pi(k)]: \Delta^{k} \rightarrow \Delta^{n}$ for the affine map that extends the map $\pi$ on the vertices. 
Definition 3.1 (symmetrisation map). The symmetrisation map

$$
\operatorname{symm}_{n}: C_{n}(X ; \mathbb{R}) \rightarrow C_{n}(X ; \mathbb{R})
$$

is the $\mathbb{R}$-linear map defined on each singular $n$-simplex $\sigma$ as

$$
\operatorname{symm}_{n}(\sigma):=\frac{1}{(n+1) !} \sum_{\pi \in \Sigma_{n+1}} \operatorname{sgn}(\pi) \cdot \sigma \circ \Delta(\pi) .
$$

Lemma 3.2 ([FM11, Lemma 2.6]). The symmetrisation map symm. is a chain map $C_{\bullet}(X ; \mathbb{R}) \rightarrow C_{\bullet}(X ; \mathbb{R})$ that is chain homotopic to the identity. Moreover, for all $c \in C_{n}(X ; \mathbb{R})$, we have

$$
\left\|\operatorname{symm}_{n}(c)\right\|_{1} \leq\|c\|_{1} .
$$

For us, the key observation is that symmetrisation enforces normalisation on cycles:

Lemma 3.3 (normalisation via symmetrisation).

(1) For all $j \in\{0, \ldots, n\}$, we have

$$
\partial_{j} \circ \operatorname{symm}_{n}=(-1)^{j} \cdot \partial_{0} \circ \operatorname{symm}_{n} .
$$

(2) In particular: If $c \in C_{n}(X ; \mathbb{R})$ is a cycle, then $\partial_{j}\left(\operatorname{symm}_{n}(c)\right)=0$ for all $j \in\{0, \ldots, n\}$.

Proof. Ad 1. Using the cyclic permutation $\tau_{j}:=\left(\begin{array}{l}j-1 \ldots 1 \\ j\end{array}\right) \in \Sigma_{n+1}$, we can re-write $\partial_{j} \circ \operatorname{symm}_{n}$ as follows: Each permutation $\pi \in \Sigma_{n+1}$ satisfies

$$
\begin{aligned}
\Delta(\pi) \circ \iota_{j}^{n} & =[\pi(0), \ldots, \pi(j-1), \pi(j+1), \ldots, \pi(n)] \\
& =\left[\pi \circ \tau_{j}(1), \ldots, \pi \circ \tau_{j}(j), \pi \circ \tau_{j}(j+1), \ldots, \pi \circ \tau_{j}(n)\right] \\
& =\Delta\left(\pi \circ \tau_{j}\right) \circ \iota_{0}^{n} .
\end{aligned}
$$

Therefore, for all singular $n$-simplices $\sigma$ on $X$ we have

$$
\begin{aligned}
\partial_{j} \circ \operatorname{symm}_{n}(\sigma) & =\frac{1}{(n+1) !} \sum_{\pi \in \Sigma_{n+1}} \operatorname{sgn}(\pi) \cdot \sigma \circ \Delta(\pi) \circ \iota_{j}^{n} \\
& =(-1)^{j} \cdot \frac{1}{(n+1) !} \sum_{\pi \in \Sigma_{n+1}} \operatorname{sgn}\left(\pi \circ \tau_{j}\right) \cdot \sigma \circ \Delta(\pi) \circ \iota_{j}^{n} \\
& =(-1)^{j} \cdot \frac{1}{(n+1) !} \sum_{\pi \in \Sigma_{n+1}} \operatorname{sgn}\left(\pi \circ \tau_{j}\right) \cdot \sigma \circ \Delta\left(\pi \circ \tau_{j}\right) \circ \iota_{0}^{n} \\
& =(-1)^{j} \cdot \frac{1}{(n+1) !} \sum_{\eta \in \Sigma_{n+1}} \operatorname{sgn}(\eta) \cdot \sigma \circ \Delta(\eta) \circ \iota_{0}^{n} \\
& =(-1)^{j} \cdot \partial_{0} \circ \operatorname{symm}_{n}(\sigma) .
\end{aligned}
$$

Ad 2. As symm. is a chain map (Lemma 3.2), if $c \in C_{n}(X ; \mathbb{R})$ is a cycle, then $\operatorname{symm}_{n}(c)$ is a cycle, and in combination with the first part we see that

$$
\begin{aligned}
0 & =\partial\left(\operatorname{symm}_{n}(c)\right)=\sum_{j=0}^{n}(-1)^{j} \cdot \partial_{j}\left(\operatorname{symm}_{n}(c)\right)=\sum_{j=0}^{n}(-1)^{2 j} \cdot \partial_{0}\left(\operatorname{symm}_{n}(c)\right) \\
& =(n+1) \cdot \partial_{0}\left(\operatorname{symm}_{n}(c)\right) .
\end{aligned}
$$

Therefore, $\partial_{0}\left(\operatorname{symm}_{n}(c)\right)=0$. Applying the first part once more shows that $\partial_{j}\left(\operatorname{symm}_{n}(c)\right)=0$ for all $j \in\{0, \ldots, n\}$. 
3.2. Proof of Proposition 1.2. We already know that $\|\alpha\|_{1} \leq\|\alpha\|_{1}^{\text {norm }}$ for every $\alpha \in H_{n}(X ; \mathbb{R})$. Let us prove the opposite inequality. Let $c \in C_{n}(X ; \mathbb{R})$ be a cycle representing $\alpha \in H_{n}(X ; \mathbb{R})$. Then, we can consider $\operatorname{symm}_{n}(c) \in$ $C_{n}(X ; \mathbb{R})$. By Lemma 3.2 we know that $\operatorname{symm}_{n}(c)$ is homologous to $c$ and satisfies $\left\|\operatorname{symm}_{n}(c)\right\|_{1} \leq\|c\|_{1}$. Moreover, Lemma 3.3 implies that $\operatorname{symm}_{n}(c)$ is normalised. This shows that

$$
\|\alpha\|_{1}^{\text {norm }} \leq\left\|\operatorname{symm}_{n}(c)\right\|_{1} \leq\|c\|_{1} .
$$

Taking the infimum over all cycles representing $\alpha$ completes the proof.

\section{REFERENCES}

[CC20] A. Connes and C. Consani, $\overline{\operatorname{Spec} \mathbb{Z}}$ and the Gromov norm, Theory Appl. Categories 35 (2020), no. 6, 155-178.

[FM11] K. Fujiwara and J. K. Manning, Simplicial volume and fillings of hyperbolic manifolds, Algebr. Geom. Topol. 11 (2011), 2237-2264.

[Gro82] M. Gromov, Volume and bounded cohomology, Publ. Math. Inst. Hautes Études Sci. 56 (1982), 5-99.

Fakultät für Mathematik, Universität Regensburg, Regensburg, Germany E-mail address: clara.loeh@ur.de

Fakultät für Mathematik, Universität Regensburg, Regensburg, Germany E-mail address: marco.moraschini@ur.de 